\title{
Combined cART including Tenofovir Disoproxil, Emtricitabine, and Dolutegravir has potent therapeutic effects in HIV-1 infected humanized mice
}

\author{
Matthew Weichseldorfer ${ }^{1}$, Yvonne Affram ${ }^{1,6}$, Alonso Heredia ${ }^{1,2}$, Zahra Rikhtegaran-Tehrani ${ }^{1}$, \\ Mohammad M. Sajadi ${ }^{1,2}$, Sumiko P. Williams ${ }^{1}$, Yutaka Tagaya ${ }^{1,2}$, Francesca Benedetti ${ }^{1,3}$, Habib O. Ramadhani ${ }^{1}$, \\ Frank Denaro ${ }^{4}$, Arshi Munawwar ${ }^{1}$, Joseph Bryant ${ }^{1}$, Davide Zella ${ }^{1,3}$, Marvin Reitz ${ }^{1}$, Fabio Romerio ${ }^{1,2,7}$ and \\ Olga S. Latinovic ${ }^{1,5^{*}}$
}

\begin{abstract}
HIV-1 reservoirs persist in the presence of combined antiretroviral therapy (CART). However, CART has transformed HIV-1 infection into a chronic disease marked by control of HIV-1 viral load and mortality reduction. Major challenges remain, including viral resistance upon termination of CART and persistence and identification of tissue distribution of HIV-1 reservoirs. Thus, appropriate animal models that best mimic HIV-1 pathogenesis are important, and the current study complements our previously published validation of the CD34+ hematopoietic humanized mouse model for this purpose. Here we analyze viral suppression using the recently developed combination of antiretrovirals that include Tenofovir Disoproxil (TDF), Emtricitabine (FTC), and Dolutegravir (DTG), a choice based on recent clinical outcomes showing its improved antiretroviral potency, CD4+ T cell preservation, tolerability, and prevention of viral drug resistance compared to that of previous regimens. We used quantitative Airyscan-based super resolution confocal microscopy of selected mouse tissues. Our data allowed us to identify specific solid tissue reservoirs of human T cells expressing the HIV-1 core protein p24. In particular, lymph node, brain, spleen, and liver were visualized as reservoirs for residual infected cells. Marked reduction of viral replication was evident. Considering that detection and visualization of cryptic sites of HIV-1 infection in tissues are clearly crucial steps towards HIV-1 eradication, appropriate animal models with pseudo-human immune systems are needed. In fact, current studies with humans and non-human primates have limited sample availability at multiple stages of infection and cannot easily analyze the effects of differently administered combined antiretroviral treatments on multiple tissues. That is easier to manage when working with humanized mouse models, although we realize the limitations due to low human cell recovery and thus the number of cells available for thorough and comprehensive analyses. Nonetheless, our data further confirm that the CD34+ humanized mouse model is a potentially useful pre-clinical model to study and improve current anti-HIV-1 therapies.
\end{abstract}

Keywords: HIV/AIDS pathogenesis, Hu-mouse models, Antiretroviral therapies

*Correspondence: olatinovic@ihv.umaryland.edu

${ }^{1}$ Institute of Human Virology, School of Medicine, University of Maryland, Baltimore, MD 21201, USA

Full list of author information is available at the end of the article

\section{Background}

HIV-1 can establish a stably integrated, non-productive latent state of infection of individual cells, mainly in long lived $\mathrm{CD} 4+\mathrm{T}$ cells that are maintained by homeostatic original author(s) and the source, provide a link to the Creative Commons licence, and indicate if changes were made. The images or other third party material in this article are included in the article's Creative Commons licence, unless indicated otherwise in a credit line to the material. If material is not included in the article's Creative Commons licence and your intended use is not permitted by statutory regulation or exceeds the permitted use, you will need to obtain permission directly from the copyright holder. To view a copy of this licence, visit http://creativecommons.org/licenses/by/4.0/. The Creative Commons Public Domain Dedication waiver (http://creativeco mmons.org/publicdomain/zero/1.0/) applies to the data made available in this article, unless otherwise stated in a credit line to the data. 
proliferation [1, 2]. Even with the evident success of cART [3], the latent existing stable reservoirs are prime barriers to HIV-1 cure. Although cART effectively suppresses HIV-1 replication, the persistent latent reservoirs of myeloid $[4,5]$ and $\mathrm{T}$ cells in patients are existing issues towards the goal of HIV-1 eradication [3, 6-8]. HIV-1 can also replicate in brain microglial cells, which persist despite cART $[9,10]$. Honeycutt et al. [11] reported that integrated HIV-1 DNA is present in human bone marrow and spleen macrophages even after cART treatment, and that mice with only human myeloid cells allow persistent infection in macrophages during cART in vivo.

Mechanisms of latency in CD4 $+\mathrm{T}$ cells in vivo are still not fully understood. In addition, better characterization of the tissues that contain cells hosting persistent virus as well as development of novel, more sensitive assays for total DNA analyses are needed for viral eradication studies [12, 13]. Since transformed cell lines used to study HIV-1 latency do not mimic the quiescent cellular environment of primary latently infected cells in vivo, humanized murine models have been proposed for characterization of HIV-1 reservoirs [14-16]. These models provide a platform for testing strategies to eliminate HIV-1 latent reservoirs and prevent viral rebound. Appropriate humanized models require proper humanization procedures of the animals as well as the use of immunodeficient recipient mouse strains that harbor various mutations [17]. An example is the SCID mouse $[18,19]$, in which a mutation in the catalytic polypeptide subunit $\left(P r k d c^{s c i d}\right)$ of the gene that encodes the DNAactivated protein kinase (DNA-PK) prevents efficient DNA repair required for $T$ and $B$ cell receptor rearrangement. This in turn results in a lack of circulating $B$ and $T$ lymphocytes. One use of such models (with immune features translatable to human) is to provide comprehensive information on different tissues during oral cART therapy, which is obviously not feasible in infected humans.

The small number of latently infected cells $(\sim 1$ in $1 \times 10^{6}$ resting $\mathrm{CD} 4+\mathrm{T}$ cells) and lack of surface latency markers make detection of HIV-1 reservoirs after antiretroviral treatment very challenging [20]. cART refractory latent reservoirs are the primary reason for the rapid virus rebound that occurs when latent cells become reactivated in humans [21], and their presence precludes complete eradication with the cART options available today. Additionally, cART is further unable to fully suppress HIV-1 replication due to poor drug penetration into certain tissues, such as the central nervous system [22].

Our previous study laid a foundation for the use of HIV-1 infected hu-NSG mice to test the effects of cART, alone or in combination with CCR5 targeting drugs, on productive HIV-1 viral replication [23]. We showed that the hu-NSG model mimics key aspects of HIV-1 pathogenesis in vivo, with HIV-1 reservoirs that are sequestered from the immune system and antiretroviral drugs. The current study looks at a recently developed cART cocktail, consisting of Tenofovir Disoproxil (TDF), Emtricitabine (FTC), and Dolutegravir (DTG) [24], using the same model and methodology as our previous studies to validate its efficacy in a humanized mouse model. In treatment-experienced patients, cART regimens based on once-daily DTG showed greater viral suppression when compared to twice-daily RAL (71\% DTG versus $64 \%$ RAL) $[25,26]$. McAllister et al. showed that DTG with TDF and FTC is a well-tolerated option for once daily postexposure in MSM [27]. In addition, DTG exhibits a higher barrier to resistance than RAL. It has a low interaction potential, so there are no food restrictions [28-30]. Based on the most recent Guidelines for the Use of Antiretroviral Agents in Adults and Adolescents with HIV-1 [31], DTG has become a recommended component in combined and recommended initial regiments for most people with HIV-1.

The hu-NSG model is an effective and valuable surgeryfree in vivo system for evaluation of HIV-1 replication, suppression, and dynamic therapy response. In addition, this model ensures that the number of available CD4+ T cells is comparable to that of HIV-1 patients under cART therapy [18]. This study allows more thorough analyses of a set of samples not readily available from human subject studies.

\section{Materials and methods}

NSG newborn mouse engraftment with human cord blood CD34+ cells

Newborn animals were transplanted with $1 \times 10^{5}$ human CD34+ hematopoietic stem cells obtained from umbilical cord blood as previously described [14, 18, 23, 32] (Lonza, donor 28753, Cat No. \#2C-101). Newborns 3-4 days old were irradiated with 100 cGy and injected intrahepatically with CD34+ cells within $3 \mathrm{~h}$ after irradiation. CD34+ cells were thawed and assessed for viability before injections. Animals were housed under pathogenfree conditions at the IHV Animal Facility, School of Medicine, University of Maryland (SOM UM) Baltimore, Maryland. All experimental protocols were in accordance with the NIH guide for the care and use of laboratory animals and approved by the SOM UM IACUC. Twenty weeks after CD34+ HSC transplantation, mice were selected based on the expansion of human CD45+ cells with $\mathrm{T}>\mathrm{B}$ cells $(\mathrm{CD} 3+$ cell numbers higher than $\mathrm{CD} 19+$ cell numbers), as judged by flow cytometry. Animals with less than a minimum of $20 \%$ human CD3+ T cells were not selected for this study [23, 32, 33]. The 13 mice selected were divided into three experimental groups: 8 
to be infected intraperitoneally with $10,000 \mathrm{TCID}_{50 \%}$ of HIV-1 BaL (200 $\mu \mathrm{L}$ total volume per mouse) at around 20 weeks of age, of which 5 mice were to be treated with oral cART for 17 weeks, and 5 mice to remain uninfected as a control group. HIV-1 BaL, an R5 replication competent virus that contains most of the BaL env gene in an HIV-1 IIIB backbone, titer $1 \times 10^{5} / \mathrm{mL}$ [34], was used to infect 8 mice. Viral stocks were provided by the $\mu$ Quant Core Lab, IHV SOM UM. The NIH regulation [35] mandating inclusion of both mouse genders in experimental groups was respected; each experimental group had sex ratios of 1:1. To monitor graft versus host disease (GVHD) that could interfere with the experimental outcome, the animals were closely observed for hair and weight loss, as they are the main indications of GVHD. No hair changes or weight reduction higher than $10 \%$ were observed. Mice were euthanized at the end of week 20 according to the IACUC protocol regulations.

\section{Treatment with antiretroviral drugs (CART)}

Doses for standard cART were chosen based on the published therapeutic efficacy in chronic HIV-1 infection in humans $[36,37]$ adjusted for mouse body weights. Food pellets (Mod TestDiet) containing cART [a mixture of Tenofovir Disoproxil (TDF), Emtricitabine (FTC) and Dolutegravir (DTG)] were administered to mice daily for 17 weeks. Cylindrical pills were manufactured as single doses of $60 \mathrm{mg}$ TDF, $60 \mathrm{mg}$ FTC, and $48 \mathrm{mg}$ DTG per $\mathrm{kg}$ of food. Pellets also contained antibiotic (amoxicillin, $0.12 \%$ ) and were irradiated before use. Food pellets containing cART mix were orally administered daily to each mouse. Mice were monitored for drug intake and given regular food pellets after finishing the medicated pellets. Mice were weighed twice per week and monitored for fur loss or change of behavior twice daily. Fresh water was changed every 2 days and always available ad libitum.

\section{Staining procedures for confocal microscopic visualization} of human lymphocytes and HIV-1 structural protein, p24 Primary PBMCs isolated from donor whole blood were cultured and activated from frozen stocks for 3 days prior to infection, then split into infected and non-infected groups, with the infected group being cultured with HIV $\mathrm{BaL}(\mathrm{MOI}=0.00075)$ for $3 \mathrm{~h}$ at $37{ }^{\circ} \mathrm{C} 5 \% \mathrm{CO}_{2}$. Afterwards, media was replaced and both groups cultured separately for 1 week. Infected and non-infected primary cells were stained using rabbit $\alpha-C D 4$ (Invitrogen, Cat. MA5-16338) primary antibody at a 1:25 dilution followed by goat $\alpha$-rabbit AF647 (Abcam, Cat. ab150079) secondary antibody at a 1:250 dilution for $45 \mathrm{~min}$ each at $4{ }^{\circ} \mathrm{C}$. Cells were fixed and permeabilized with Foxp3 Fixation and Permeabilization Buffer (Invitrogen, Cat. 00-552300) for 45 min at $4{ }^{\circ} \mathrm{C}$, then stained using mouse $\alpha$-p 24
FITC (Beckman Coulter, Cat. 6604665) at 1:25 for $45 \mathrm{~min}$ at $4{ }^{\circ} \mathrm{C}$ followed by $50 \mu \mathrm{g} / \mathrm{mL}$ DAPI for $1 \mathrm{~min}$. Cells were imaged in 1\% PFA diluted in PBS. Controls were stained without rabbit $\alpha-C D 4$ primary and/or with mouse IgG1 Alexa Fluor 488 (Biolegend, Cat. 400134) in place of $\alpha$-p24 FITC. HIV-1 negative and control cells showed no positive staining (not shown).

For tissue imaging, slides were warmed $\left(45^{\circ} \mathrm{C}\right)$ for at least $10 \mathrm{~min}$ to remove paraffin, then submerged in xylene. Containers with slides were placed into dehydration buckets containing 100\% ethanol and incubated for a few minutes. The procedure was repeated several times with lower concentrations of ethanol, and the slides were then incubated at $95{ }^{\circ} \mathrm{C}$ in Dako Target Retrieval Solution (Dako, Cat No. S2368) for $20 \mathrm{~min}$ and cooled per the manufacturer's instruction. Blocking was overnight at $4{ }^{\circ} \mathrm{C}$ with $20 \%$ BSA in $1 \times$ PBS. Surface marker staining used 1:25 $\alpha$-CD4 Alexa Fluor 488 (Stemcell Technologies, Cat. 60016AD), 1:25 primary antibody $\alpha-C D 45$ (Leica BioSystems, Cat. NCL-L-LCA), or 1:25 primary antibody $\alpha$-CD68 (Dako, Cat. M0814) for $1 \mathrm{~h}$ at room temperature (RT). Slides stained against CD45 or CD68 were then incubated with 1:250 secondary antibody $\alpha$-mouse IgG $(\mathrm{H}+\mathrm{L})$ DyLight 488 (Vector, Cat. DI-2488) for $1 \mathrm{~h}$ at RT. Slides were permeabilized with $0.1 \%$ Triton X-100 in $1 \times$ PBS at RT for 10 min, blocked overnight with $20 \%$ BSA in $1 \times$ PBS at $4{ }^{\circ} \mathrm{C}$, and labeled with 1:25 primary antibody $\alpha$-p24 (Sino, Cat No. 40243-RP01) for $1 \mathrm{~h}$ at RT, followed by secondary antibody labeling with 1:250 $\alpha$-rabbit Alexa Fluor 647 (abcam, Cat No. ab150079) for $1 \mathrm{~h}$ at RT. Slides were DAPI stained using $50 \mu \mathrm{g} / \mathrm{mL}$ DAPI for $2 \mathrm{~min}$ and treated with $1 \times$ True Black Blocker (Biotium, Cat No. 23007) for $30 \mathrm{~s}$. Slides were cover slipped with Vectashield Mounting Media (Vector, Cat No. H-1000) prior to imaging. Control samples were stained with the same conditions described above, and for additional controls, HIV-1 negative cells/tissues were stained with DAPI and secondary antibody only (not shown).

\section{Image acquisition}

Confocal images of cell-associated fluorescence were acquired using the Zeiss LSM 800 confocal system (Carl Zeiss Microscopy, Germany) via the Airyscan super resolution mode. Three laser lines, $405 \mathrm{~nm}$ (blue, for nuclei), $488 \mathrm{~nm}$ (green, for leukocyte surface antigens [CD45 or CD4 in liver, LN, and spleen; CD68 in brain] and $647 \mathrm{~nm}$ (red, for HIV-1 protein, p24) were used. Blue, green, and red signals were separated by a quad DAPI/FITC/ TRITC/Cy5 dichroic beam splitter and further acquired using a Gasp detector. A Plan-Apochromat 63x/1.4 Oil DIC objective was used to visualize multi-color labelled cell/tissue samples. ZEN Blue 2.3 software (Carl Zeiss Microscopy, Germany) was used to generate original 
images. All images were acquired under the same instrument settings. Signal to noise ratio was accounted for by averaging data all images acquired using the same gain offset, detector, and laser excitation power. Saturated signal was avoided by using the software-controlled range for a minimum pixel saturation.

\section{Peripheral blood viral load}

Mice were periodically bled retro-orbitally. RNA was extracted from plasma using a QIAamp Viral RNA Mini Kit (QIAGEN, Cat. 52904) as per the manufacturer's instructions. HIV-1 RNA was converted to cDNA using a SuperScript III First-Strand Synthesis SuperMix Kit (Invitrogen 18080-400) as per the manufacturer's instructions. HIV-1 cDNA was amplified and quantified using qPCR with the following protocol: a single cycle at $50{ }^{\circ} \mathrm{C}$ for $2 \mathrm{~min}$, a single cycle at $95{ }^{\circ} \mathrm{C}$ for $15 \mathrm{~min}, 40$ cycles at $94{ }^{\circ} \mathrm{C}$ for $15 \mathrm{~s}, 58{ }^{\circ} \mathrm{C}$ for $30 \mathrm{~s}$, and $72{ }^{\circ} \mathrm{C}$ for $30 \mathrm{~s}$, followed by a single cycle at $72{ }^{\circ} \mathrm{C}$ for $30 \mathrm{~s}$ using a BioRad LightCycler and BioRad iQ5 software. HIV-1 RNA data were graphed using GraphPad Prism 9 for each mouse ( $n=3$ per treatment; randomly chosen), along with an average for each treatment.

\section{DNA standard curve}

To generate a standard to quantify HIV-1 DNA copies via qPCR, HIV-1 gag amplicons were cloned using a TOPO ${ }^{\mathrm{TM}}$ TA Cloning ${ }^{\mathrm{TM}}$ Kit (Invitrogen, Cat. 450640), transfected into One Shot ${ }^{\circledR}$ TOP10 E. coli (Invitrogen, Cat. C404004), grown on an LB agar plate containing $50 \mu \mathrm{g} / \mathrm{mL}$ kanamycin, and DNA extracted from overnight cultures using a QIAprep ${ }^{\circledR}$ Spin Miniprep Kit (QIAGEN, Cat. 27104). Plasmid DNA was sequenced to determine HIV-1 gag integrity and serially diluted for standards [38].

Standards were qPCR amplified alongside mouse tissue DNA samples using primers specific to either HIV-1 gag (at 30,000, 3000, 300, 30, and 3 standard copies) or human $\beta$-globin (at 100,000,10,000,1000, 100, and 10 standard copies). Cycle thresholds were plotted against initial template quantity on a log scale to create standard curves [38]. DNA sample thresholds were plotted against the standard curves to quantify DNA copy number.

\section{HIV-1 gag DNA quantification in tissue.}

Viral DNA loads in mouse tissue were quantified by a qPCR assay measuring total viral DNA (integrated and unintegrated, linear, and circular forms). DNA was extracted from mouse tissue (brain, liver, spleen, and lymph node) using a QIAGEN DNeasy ${ }^{\circledR}$ Blood and Tissue Kit (QIAGEN, Cat. 69506) and analyzed using the QuantStudio 3 PCR system (Applied Biosystems) with $10 \mu \mathrm{L}$ of genomic DNA in each sample. Samples were analyzed along with the set of standards for both HIV-1 gag DNA and human $\beta$-globin DNA in 96-well plates in triplicate. DNA copy numbers were quantified with the QuantStudio 3 PCR system using the standard TaqMan protocol. This consisted of a single cycle at $50{ }^{\circ} \mathrm{C}$ for 5 min, a single cycle at $95^{\circ} \mathrm{C}$ for $10 \mathrm{~min}$, and 45 cycles at $95{ }^{\circ} \mathrm{C}$ for $15 \mathrm{~s}$ and $60^{\circ} \mathrm{C}$ for one minute. All qPCR experiments were repeated three times for reproducibility. Unknown HIV-1 gag or human $\beta$-globin DNA copy numbers were determined by extrapolation from the known standard DNA copy numbers for HIV-1 gag DNA or human $\beta$-globin. We expressed HIV-1 gag copies as copies per $10^{6}$ cells. All statistics were calculated using GraphPad Prism 9.

\section{Results}

Hu-mouse tissue samples were selected based upon our previous study [23] where we demonstrated successful CD34+ cell engraftment in NSG mice, verified by flow cytometry identification of human CD45+, CD4+, and CD8 $+\mathrm{T}$ cells. Previous data showed an average of $64 \%$ engraftment of $\mathrm{CD} 3+\mathrm{T}$ cells, $38 \%$ engraftment of CD4+ T cells, $10 \%$ engraftment of CCR5 + T cells, and $23 \%$ engraftment of CD8 $+\mathrm{T}$ cells, on average, within the total population of $\mathrm{CD} 45+$ cells [23, 32]. Flow cytometry data for human cells present in hu-mice were normalized with respect to CD45+ cell numbers. Viral load measurements from infected mice confirmed stable, productive HIV-1 infection reaching a maximum of $5 \times 10^{5}$ to $5 \times 10^{6}$ copies $/ \mathrm{mL}$ at around week 3 post-infection. This load was generally maintained through week 20 post-infection [14, 18, 23].

The timeline is represented in Fig. 1. At 20 weeks prior to viral challenge, 13 new-born NSG mice were irradiated ( $100 \mathrm{cGy}$ ), then engrafted with $1 \times 10^{5}$ human CD34+ hematopoietic stem cells each and evaluated 20 weeks later for engraftment by flow cytometry, with the criteria that $\mathrm{CD} 3+$ cell numbers must be greater than $\mathrm{CD} 19+$ cell numbers. Eight mice were infected intraperitoneally with 10,000 TCID $_{50}$ HIV-1 BaL [34] at week 0 (Fig. 1) with 5 mice remaining as uninfected controls. Random mice were bled and evaluated for viral RNA, as well as human cell numbers to confirm productive HIV-1 infection (Fig. 3). At week 3, infected mice were split into an untreated group (3 mice) and a cART-treated group (5 mice). cART therapy was initiated and orally administered once daily, in the form of food pellets, until the endpoint at week 20 where they were evaluated for viral RNA (3 randomly chosen mice per treatment group) and intracellular DNA. 


Week -20
Engraftment
FuCD34+Cells
Fig. 1 Timeline of experimental steps. Newborn mice were irradiated and injected with human CD34+ hematopoietic stem cells. After 20 weeks,
engrafted mice were infected with 10,000 TCID 50\% HIV-1 BaL and a subset treated with cART 3 weeks post-infection. This subset of HIV-1
infected mice received oral cART containing Dolutegravir (DTG), Tenofovir Disoproxil (TDF), and Emtricitabine (FTC) for 17 weeks. After 20 weeks
post-infection, mice were euthanized, and organs collected for qPCR and imaging experiments. Total DNA was quantified using qPCR in all four
collected tissues, and infection sites mapped via p24 imaging in all four collected tissues using super resolution Airyscan imaging

Visualization of HIV-1 p24 protein in HIV-1 infected hu-mice to demonstrate productive HIV-1 infection To confirm CD34+ cell engraftment and productive HIV-1 infection, we analyzed HIV positive and HIV negative mouse livers, spleens, and LN tissues for the presence of human $\mathrm{T}$ cells and for expression of viral p24 by Airyscan confocal microscopy before proceeding with functional assays. In Fig. 2A, a single $\mathrm{T}$ cell infected

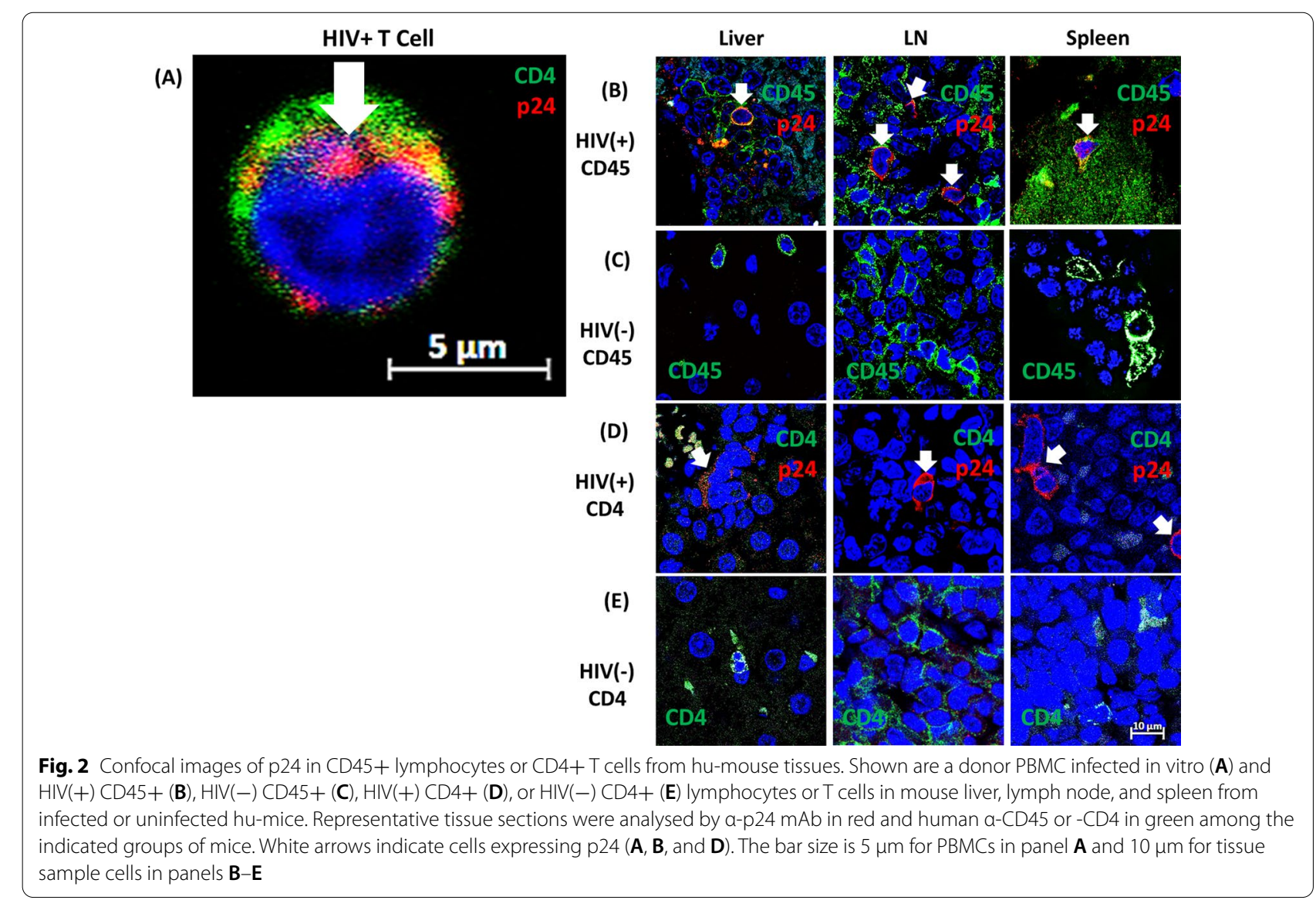


in vitro represents the positive control for cytoplasmic p24 (red fluorescence) as well as $\alpha$-huCD4 Ab (green fluorescence). The nucleus is blue from the nuclear dye DAPI. In vitro optimization of the staining procedure was applied to the following tissue staining experiments with 754.34 PE mol/cell; PE being the fluorophore for FACS evaluation of surface CCR5. As shown in Fig. 2B, $\mathrm{D}$, cell-associated p24 was detectable, as indicated by white arrows, in LN, spleen, and liver of HIV-1 infected hu-mice.

We previously showed successful engraftment with huCD45+ and CD4+ T cells in peripheral blood samples via flow cytometry [23]. The best-defined HIV-1 reservoirs which persisted during the CART regimens were located inside the latently infected CD4+ T cells $[39,40]$. We initially focused on visualizing infected CD4+ T cells. However, infected huCD4+ cells were rare in solid tissues from infected mice, suggesting down-regulation of CD4 expression because of HIV infection. In contrast, LN, liver, and spleen of noninfected mice (Fig. 2E, green fluorescence) did contain huCD4+ cells, suggesting either that infection depletes these cells from solid tissue, or that CD4 is downregulated by Nef. However, CD45+ lymphocytes were present that also expressed p24, as clearly shown in Fig. 2B, demonstrating productive HIV-1 infection of lymphocytes. Figure $2 \mathrm{~B}-\mathrm{E}$ present 12 individual images [ 6 for $\mathrm{HIV}(+)$ and 6 for uninfected mice] from three different organs, stitched together via ZEN Blue 2.3 software. Similar results were obtained with intestinal and brain tissues from HIV-1(+) hu-mice (not shown). There was a major difference between the number of cells in Fig. 2D, E because human CD4+ T cells, which are depleted by HIV-1 infection, are the majority (>60\%) of human CD45+ cells in uninfected humanized mice (as previously shown by flow cytometry [23]). We also stained for infected monocytes/macrophages and microglia in brain tissue using CD68 as a marker. Both CD68+p24+ and CD68+p24- cells were evident in infected mice (Additional file 1: Figure S1).

\section{Reduction of viral RNA in peripheral blood by modified cART}

We quantified viral RNA copy numbers by qRT-PCR to demonstrate HIV-1 infection and show reduction in HIV-1 RNA copy numbers by this cART combination (Fig. 3). In HIV-1 infected samples, HIV-1 copies remained relatively consistent at approximately $10^{5}-10^{6}$ copies $/ \mathrm{mL}$ for up to 20 weeks post-infection. In infected samples treated with the modified cART, HIV-1 copies remained relatively consistent at approximately $10^{5}-10^{6}$ copies/mL until administration of cART at week 3 , at which point they rapidly dropped to undetectable and remained there for up to 20 weeks post-infection when cART treatment ended. HIV-1 negative mice had no detectable HIV-1 RNA for the entire 20 weeks, as expected. Each set of data were obtained from randomly chosen animals. The samples were assayed twice to ensure reproducibility, as shown by the black lines. The $\mathrm{p}$ values for the ratios are shown in Fig. 3. HIV $(+)$ cART $(+)$ significance compared to HIV(-) could not be calculated due to all end point values equalling zero, but the data sets are identical in their lack of HIV-1 detectability.

HIV-1 Viral Load

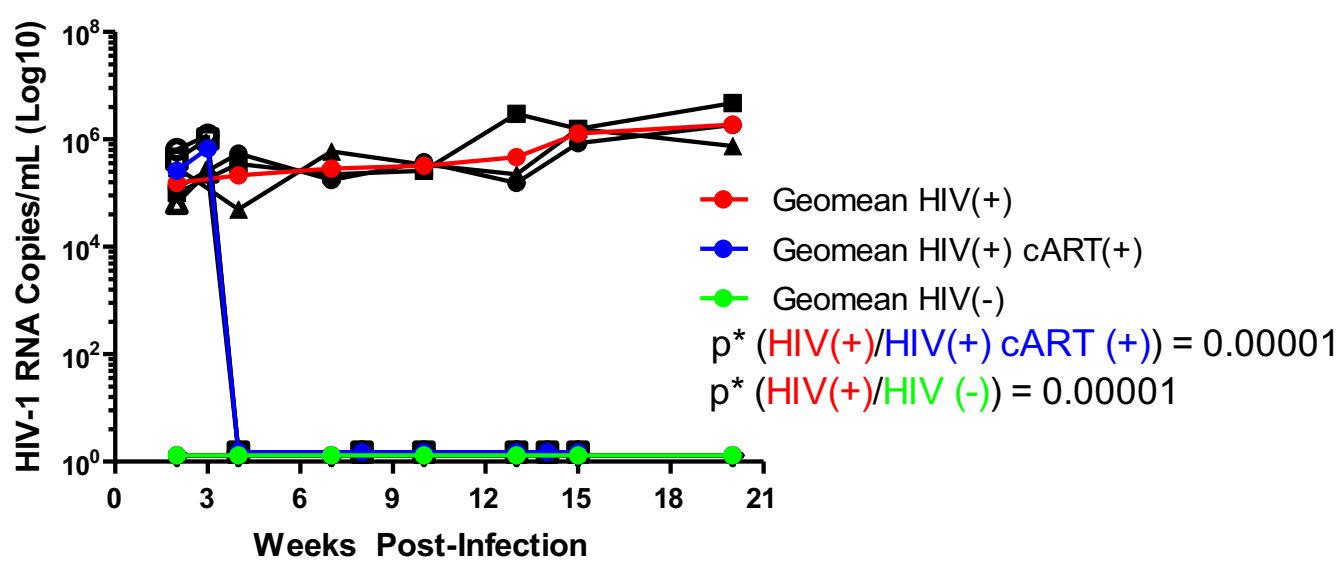

Fig. 3 Peripheral blood viral loads. Mice were bled every few weeks and RNA isolated from plasma. RNA copies/mL were calculated from qRT-PCR as described in "Materials and methods". Copies $/ \mathrm{mL}$ are graphed on a log 10 scale based on weeks post-infection. cART treatment was initiated at 3 weeks p.i. Each treatment group contained 3 mice. A color-coded relative geometric mean for each treatment group was added with HIV $(+)$ in red, $\operatorname{HIV}(+)$ CART (+) in blue, and HIV(-) in green 


\section{Reduction of viral DNA in tissue by modified CART}

We quantified viral DNA copy numbers to assess whether the new cART combination effectively decreased the viral load in solid tissues. We used a qPCR assay originally validated in primary cells [38] to quantify viral DNA in hu-mouse HIV-1 and cART treated tissues. We first compared $\mathrm{Ct}$ values of our standard derived from infected hu-mouse tissue with those from ACH.2 cells, which have a single copy of proviral DNA. Agreement of the two sets of values at different input levels showed the method to be accurate (Additional file 1: Figure S2).

Next, we quantified total viral DNA (integrated and unintegrated) in LN, liver, brain, and spleen tissue from infected hu-mice to determine the effect of the cART treatment. The standard DNA curve used in this experiment is described in detail in Additional file 1: Figure S2.
The mice were treated orally with the new cART formulation for 17 weeks total after the initial 3-week HIV-1 infection. We also collected LN, liver, brain, and spleen tissues from non-infected controls. Figure 4 shows DNA copy numbers in tissues from these three experimental groups. The first columns of data show viral DNA copy numbers in HIV-1 infected and non-treated mice, the second columns show the cART-treated mice, and the third column the uninfected mice. The infected group contained 3 experimental animals, with a male/female ratio of 2 males:1 female, infected/cART group contained 5 experimental animals, with a male/female ratio of 3 males: 2 females. The uninfected group contained 5 experimental animals ( 3 males:2 females).
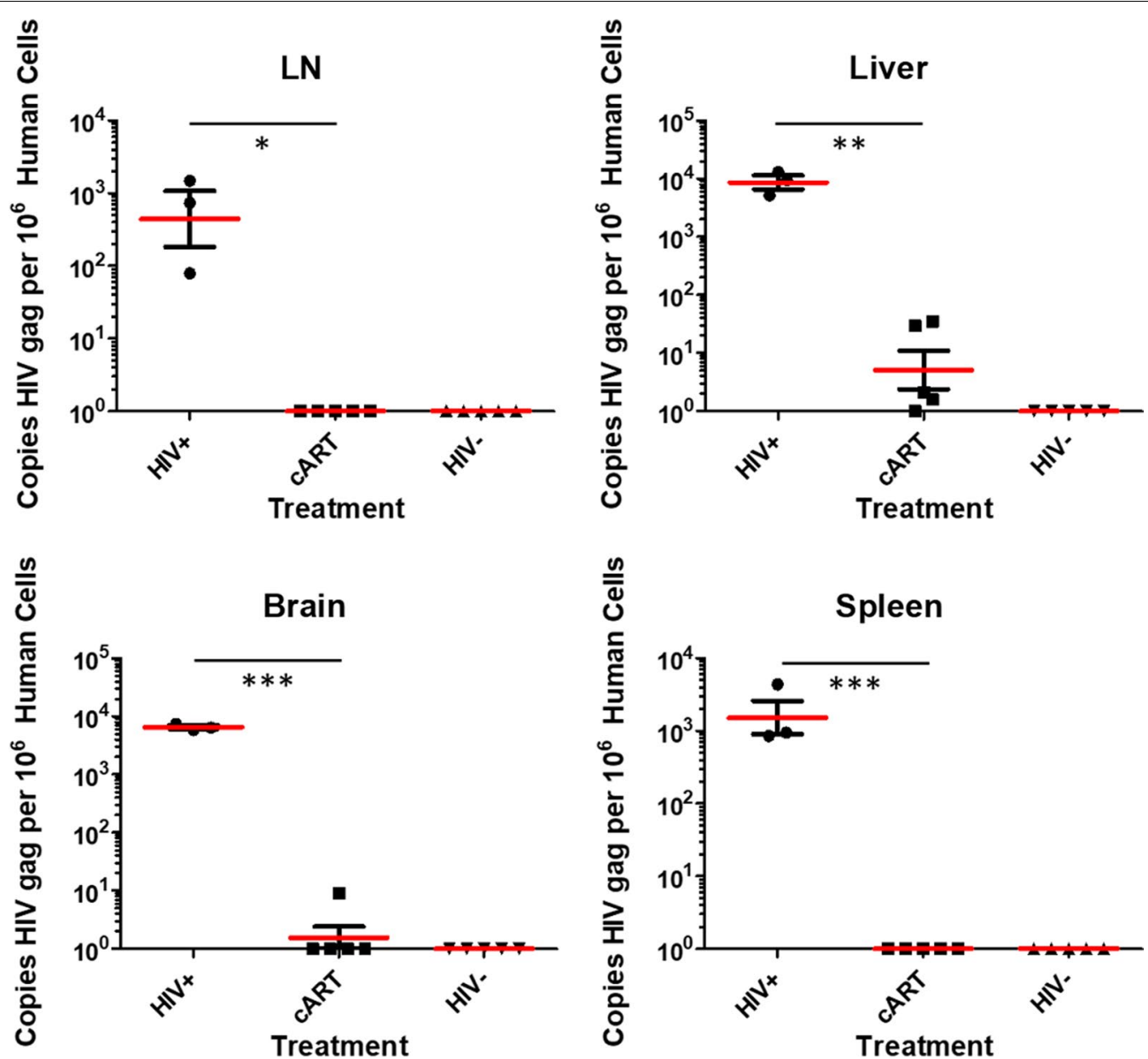

Fig. 4 Viral DNA levels in tissues corresponding to visualized productive HIV-1 infection sites in Fig. 2. The first column in each panel represents tissue samples from infected mice; the second column represents tissues from HIV-1 infected, but CART treated mice; and the third column represents tissues from uninfected mice. The first panel is from $L N$, the second panel from liver, the third panel from brain, and the fourth panel from spleen tissues. The data include groups of 3 animals for the HIV-1 positive group, and 5 different animals for the HIV- 1 positive, cART treated group and the HIV-1 negative control group. P values are $0.041,0.001,<0.001$, and $<0.001$ respectively, with the red lines indicating the average value of 3 or 5 mice per group. Copy numbers are graphed on a $\log _{10}$ scale and reported as copies per $10^{6}$ human cells. ${ }^{*} p<0.05,{ }^{* *} p<0.01,{ }^{* * *} p<0.001$ 
The data in Fig. 4 show a striking reduction of viral DNA levels in the solid tissues of mice treated with the new cART combination. The cART was most effective in the LN and spleen tissues of the hu-mice but was also quite effective in the brain and liver, although viral DNA was still detectable in those tissues. The data in Fig. 4 are from three independent experiments using the same mouse tissues per experimental group. Total viral DNA copy numbers in infected but untreated mice were highest for liver (an average of 9275.3 copies per $10^{6}$ cells). Brain, LN, and spleen contained 6547.1, 767.4, and 902.55 copies $/ 10^{6}$ human cells respectively. In contrast to infected but untreated tissue, treatment with the new cART showed lower viral DNA copy numbers (11.4 per $10^{6}$ cells in liver and 1.5 per $10^{6}$ cells in brain), while no viral DNA was detected in LN or spleen. Residual viral DNA in cART treated liver and brain thus supports the need for further intensification of cART. This could involve including CCR5 targeting drugs, the efficacy of which was shown by our previous results in vitro and in vivo $[23,38]$.

\section{Discussion}

This study showed that the cART treatment used in our experiments successfully decreased HIV-1 RNA in peripheral blood and DNA numbers in HIV-1 infected hu-mouse tissues such as spleen, lymph nodes, liver, and brain. It further confirms that the hu-NSG mouse model is appropriate for preclinical testing of advanced antiretroviral therapies for their ability to reduce HIV-1 viral replication, particularly since such models provide various accessible tissues at different stages of treatment. We offer additional support for this model by showing visualized, selected sites of HIV-1 infection in different hu-mouse tissues using super resolution Airyscan confocal microscopy and a striking reduction of viral DNA copies in HIV-1 hu-mice treated with a combination of Tenofovir Disoproxil (TDF), Emtricitabine (FTC), and Dolutegravir (DTG) using real time qPCR. We selected this drug combination based on recent evidence of its superior antiviral efficacy in clinical studies $[27,28,41$, 42] compared with previous cARTs. This study could provide the basis for future studies in vivo using treatments such as nano-formulated therapies, which could include enhancement with CCR5 targeting drugs as a possibility to intensify this cART or cytostatic drugs such as tyrosine kinase inhibitors (TKIs) as adjuvants to standard cART options.

There have been several approaches to achieve sustained viral remission, including passive immunization, early initiation of CART, therapeutic vaccination, and immune modulation with clearance of HIV-1 infected cells [43]. Current cART offers sustained and durable
HIV-1 suppression [44] that is largely effective in stopping viral replication. However, to date no drug combinations have been able to fully eradicate viral reservoirs in HIV-1 patients. The ability of HIV-1 to establish latent infection causes the formation of these reservoirs in various tissues, including the central nervous system, LN, gut, and bone marrow. Two major factors favoring the existence of viral reservoirs are the longevity of latently infected cells and ineffective drug penetration into some organs (particularly the central nervous system, [45]). Detection and visualization of cryptic sites of HIV-1 infection in tissues are clearly crucial steps towards HIV-1 eradication [46] and will require appropriate animal models with pseudo-human immune systems.

The existing problem in studies with humans and nonhuman primates are the limitations on sample availability at multiple stages of infection and from differently administered combined antiretroviral treatments. That is easier to manage when working with humanized mouse models. A convenient small animal model for these types of studies is clearly necessary, although we realize that such a model will likely present limitations due to low human cell recovery and thus the number of cells available for thorough and comprehensive analyses. Sample size is somewhat further limited due to mouse body size (20-22 g), but these issues can be addressed by advanced imaging and more sensitive qPCR techniques [47]. However, the main advantage in working with hu-mice is the accessibility to various organ tissues under different treatment options and different therapy timing. Interestingly, Satou et al. demonstrated that there is a dynamic of clonal expansion of HIV-1 infected cells in hu-mice which makes the hu-mouse models more attractive [48]. Lastly, there appears to be less heterogeneity in the humanized mice than in infected individuals, as previous studies have shown thousands of integration sites in humans [48] (although the number of patients analyzed was small). This is relevant to consider, because there are differences in terms of founder virus sequences, routes of initial infections, and duration of HIV-1 infection and treatments that can affect clonal expansion of infected cells and their tissue distribution.

With these considerations in mind, we have used a humouse model for this type of antiretroviral treatment study and determined to what extent the new combination of cART drugs reduces, depletes, or prevents the establishment of viral reservoirs. A great advantage of this human hematopoietic stem cell-engrafted NOD/ SCID/IL2R $\gamma$ null (NSG) model is the shorter time for human cell reconstitution compared with other humouse models [19].

We first visualized HIV-1 infection by showing expression of p24 in selected mouse tissues (Fig. 2 and 
Additional file 1: Figure S1). We next measured plasma viral loads to demonstrate cART efficacy (Fig. 3) and quantified total viral DNA copy numbers in selected solid mouse tissues. Both plasma viral RNA and viral DNA in tissues were greatly reduced by this cART. By super resolution confocal imaging, which allows more precise visualization of p24 within HIV-1 positive cells, we showed multiple sites of productive HIV-1 infection, allowing us to assess HIV-1 suppression by the new cART combination [14, 18]. HIV-1 viral replication, as judged by HIV-1 DNA copies in LN, liver, spleen, and brain, were substantially reduced or eliminated by treatment with this combination for 17 weeks.

Significant efforts have been made to eradicate HIV-1 reservoirs present as latently infected $\mathrm{CD} 4+\mathrm{T}$ cell subsets within solid tissues [14]. In this study, as judged by total intracellular DNA copy numbers in HIV-1 infected hu-mice, the new current cART treatment is effective in inhibition of viral replication, which reduces the establishment of new reservoirs. Our current study further demonstrates the utility of this hu-mouse model to analyze in vivo HIV-1 infection kinetics and to identify HIV-1 productive tissue by confocal microscopy and functional assays, although one shortcoming is a lack of the full human immune cell spectrum. Additional hu-mouse studies are needed to address how to inhibit clonal expansion of HIV-1 infected cells due to $\mathrm{T}$ cell homeostasis.

Brain and liver tissues appeared to contain higher apparent viral loads than LN, possibly because they contained a higher proportion of infected cells, rather than a higher number of infected cells. LN had a higher copy number, but a lower copy/cell number. LN are highly populated with human cells, and due to a relatively short infection period, the infection may not have been able to spread among such a large population before samples were collected and analyzed. The lack of total HIV-1 DNA suppression in the liver and brain tissues of HIV-1 infected hu-mice treated with this combination of cART drugs (Fig. 4), indicates the need for further cART drug intensification strategies, perhaps by inclusion of CCR5 targeting drugs.

\section{Supplementary Information}

The online version contains supplementary material available at https://doi. org/10.1186/s12967-021-03120-w.

Additional file 1: Figure S1. Confocal images of p24 in CD68+ macrophages from hu-mouse brain. Shown are an HIV(+) CD68+ (A) and $\mathrm{HIV}(-) \mathrm{CD} 68+(\mathbf{B})$ macrophages in brain tissue from infected or uninfected hu-mice. Representative tissue sections were analysed by $a-p 24$ mAb in red and human a-CD68 mAb in green among the indicated groups of mice. White arrows indicate cells expressing p24 (A). The bar size is $10 \mu \mathrm{m}$ for tissue sample cells in panels $\mathbf{A}$ and $\mathbf{B}$. Figure $\mathbf{S 2}$. Comparison of ACH.2 and hu-mouse Plasmid DNA Standards. Threshold cycle (Ct) measured during qPCR using either total intracellular DNA from ACH.2 chronically infected cells or a plasmid that contains the HIV-1 DNA fragment that was originally isolated from an infected hu-mouse tissue. The Ct at 3000, 300, 30, and 3 copies are shown in each graph, with red bars demonstrating the average value and black bars representing standard error.

\section{Acknowledgements}

The authors thank Mr. Harry Davis from IHV Animal Facility for good care of the animals.

\section{Authors' contributions}

Conceptualization: MR and OSL; formal analysis: MW; methodology: MW, YA, ZRT, SPW, FB, FD, AM, DZ and FR; supervision: OSL; writing —original draft: OSL; writing — review and editing: MW, AH, ZRT, MMS, YT, AM, JB, MR, FR and OSL. All authors read and approved the final manuscript.

\section{Funding}

This project was funded by the internal funds of the Institute of Human Virology, University of Maryland, and School of Medicine.

\section{Availability of data}

The datasets during and/or analyzed during the current study available from the corresponding author on reasonable request.

\section{Declarations}

\section{Ethics approval and consent to participate}

Animals were housed under pathogen-free conditions at the IHV Animal Facility, School of Medicine, University of Maryland (SOM UM) (Baltimore, MD). All experimental protocols were in accordance with the $\mathrm{NIH}$ guide for the care and use of laboratory animals and approved (Protocol \#1215007) by the SOM UM IACUC.

\section{Consent for publication}

Not applicable.

\section{Competing interests}

The authors declare that they have no competing interests.

\section{Author details}

${ }^{1}$ Institute of Human Virology, School of Medicine, University of Maryland, Baltimore, MD 21201, USA. '2 Department of Medicine, School of Medicine, University of Maryland, Baltimore, MD 21201, USA. ${ }^{3}$ Department of Biochemistry and Molecular Biology, School of Medicine, University of Maryland, Baltimore, MD 21201, USA. ${ }^{4}$ Morgan State University, College of Bio Sciences, Baltimore, MD 21011, USA. ${ }^{5}$ Department of Microbiology and Immunology, School of Medicine, University of Maryland, Baltimore, MD 21201, USA. ${ }^{6}$ Department of Microbial Pathogenesis and Immunology, University of Texas A and M Health Science Center, Bryan, TX 77843, USA. ${ }^{7}$ Department of Molecular and Comparative Pathobiology, Johns Hopkins University School of Medicine, Baltimore, MD 21201, USA

Received: 20 September 2021 Accepted: 16 October 2021 Published online: 30 October 2021

\section{References}

1. Chomont N, El-Far M, Ancuta P, Trautmann L, et al. HIV reservoir size and persistence are driven by $T$ cell survival and homeostatic proliferation. Nat Med. 2009;15(8):893-900.

2. Bosque A, Famiglietti M, Weyrich AS, et al. Homeostatic proliferation fails to efficiently reactivate HIV-1 latently infected central memory CD4+ T cells. PloS Pathog. 2011;7(10):e1002288.

3. Katlama C, Deeks SG, Autran B, et al. Barriers to a cure: new concepts in targeting and eradicating HIV-1 reservoirs. Lancet. 2013. https://doi.org/ 10.1016/S0140-6736(13)60104-X. 
4. Natarajan V, Bosche M, Metcalf JA, Ward DJ, Lane HC, Kovacs JA. HIV-1 replication in patients with undetectable plasma virus receiving HAART. Lancet. 1999;353:119-20. https://doi.org/10.1016/S0140-6736(05) 76156-0.

5. Martinez-Picado J, Deeks SG. Persistent HIV-1 replication during antiretroviral therapy. Curr Opin HIV AIDS. 2016;11:417-23. https://doi.org/10. 1097/COH.0000000000000287.

6. Ho YC, Shan L, Hosmane NN, Wang J, Laskey SB, Rosenbloom DI, Lai J, Blankson JN, Siliciano JD, Siliciano RF. Replication-competent noninduced proviruses in the latent reservoir increase barrier to HIV-1 cure. Cell. 2013;155:540-51. https://doi.org/10.1016/j.cell.2013.09.020.

7. Richman DD, Margolis DM, Delaney M, Greene WC, Hazuda D, Pomerantz RJ. The challenge of finding a cure for HIV infection. Science. 2009;323:1304-7. https://doi.org/10.1126/science.1165706.

8. Margolis DM. How might we cure HIV? Curr Infect Dis Rep. 2014;16:392. https://doi.org/10.1007/s11908-014-0392-2.

9. Price RW, Brew B, Sidtis J, et al. The brain in AIDS: central nervous system HIV-1 infection and AIDS dementia complex. Science. 1988;239:586-92. https://doi.org/10.1126/science.3277272.

10. Gorry PR, Howard JL, Churchill MJ, et al. Diminished production of human immunodeficiency virus type 1 in astrocytes results from inefficient translation of gag, env, and nef mRNAs despite efficient expression of Tat and Rev. J Virol. 1999;73:352-61.

11. Honeycutt JB, Wahl A, Baker C, et al. Macrophages sustain HIV replication in vivo independently of T cells. J Clin Invest. 2016;126:1353-66. https:// doi.org/10.1172/JCl84456.

12. Swanstrom R, Coffin J. HIV-1 pathogenesis: the virus. Cold Spring Harb Perspect Med. 2012;2(12):a007443.

13. Lorenzo-Redondo R, Fryer HR, Bedford T, et al. Persistent HV-1 replication maintains the tissue reservoir during therapy. Nature. 2016;530:51-63.

14. Arainga $M$, Su H, Poluektova LY, et al. HIV-1 cellular and tissue replication patterns in infected humanized mice. Sci Rep. 2016;6:23513.

15. Dash PK, Gorantla S, Gendelman HE, et al. Loss of neuronal integrity during progressive HIV-1 infection of humanized mice. J Neurosci. 2011;31:3148-57

16. Nischan M, Gers-Huber G, Audige A, et al. Modeling HIV infection and therapies in humanized mice. Swiss Med Wkly. 2012;142:w12618.

17. Shultz LD, Brehm MA, Garcia-Martinez JV, et al. Humanized mice for immune system investigation: progress, promise and challenges. Nat Rev. 2012;12:786-98.

18. Satheesan S, Haitang L, Burnett JC, et al. HIV Replication and latency in a humanized NSG mouse model during suppressive oral combinational antiretroviral therapy. J Virol. 2018;92:e02118-e2217.

19. Weichseldorfer M, Heredia A, Reitz M, et al. Use of humanized mouse models for studying HIV-1 infection pathogenesis and persistence. J AIDS HIV Treat. 2020;2(1):23-9.

20. Sengupta S, Siliciano RF. Targeting the latent reservoir for HIV-1. Immunity. 2018;48(5):872-95.

21. Vanhamel J, Bruggemans A, Debyser Z. Establishment of latent HIV-1 reservoirs: what do we really know? J Virus Erad. 2019;5(1):3-9.

22. Gray LR, Roche M, Flynn JK, et al. Is the central nervous system a reservoir of HIV-1? Curr Opin HIV AIDS. 2014;9(6):552-8.

23. Latinovic OS, Neal LM, Tagaya Y, et al. Suppression of active HIV-1 infection in CD34+ hematopoietic humanized NSG mice by a combination of combined antiretroviral therapy and CCR5 targeting drugs. AIDS Res Hum Retrovir. 2019;35(8):718-28.

24. Cahn P, Pozniak AL, Mingrone $H$, et al. Dolutegravir versus raltegravir in antiretroviral-experienced, integrase-inhibitor-naive adults with HIV: week 48 results from the randomised, double-blind, non-inferiority SAILING study. Lancet. 2013;382:700-8.

25. Brehm TT, Franz M, Hufner A, et al. Safety and efficacy of elvitegravir, dolutegravir, and raltegravir in a real-world cohort of treatment-naïve and -experienced patients. Medicine. 2019;98(32):e167.

26. Taha H, Das A, Das S. Clinical effectiveness of dolutegravir in the treatment of HIV/AIDS. Infect Drug Resist. 2015;8:339-52.

27. McAllister JW, Towns JM, Mcnulty A, et al. Dolutegravir with tenofovir disoproxil fumarate-emtricitabine as HIV postexposure prophylaxis in gay and bisexual men. AIDS. 2017;31:1291-5

28. Raffi F, Jaeger H, Quiros-Roldan E, et al. Once-daily dolutegravir versus twice-daily raltegravir in antiretroviral-naive adults with HIV-1 infection
(SPRING-2 study): 96 week results from a randomised, double-blind, noninferiority trial. Lancet Infect Dis. 2013;13:927-35.

29. Powderly WG. Integrase inhibitors in the treatment of HIV-1 infection. J Antimicrob Chemother. 2010;65:2485-8

30 Venter EDF, Moorhouse S, Sokhela L, et al. Dolutegravir plus two different prodrugs of tenofovir to treat HIV. N Engl J Med. 2019;381(9):803-15.

31. Guidelines for the use of antiretroviral agents in adults and adolescents with HIV.

32. Arainga $M$, Edagwa $B$, Mosley $R L$, et al. A mature macrophage is a principle HIV-1 cellular reservoir in humanized mice after treatment with long acting antiretroviral therapy. Retrovirology. 2017;14:17.

33. Deruaz M, Moldt B, Le KM, Power KA, Vrbanac VD, Tanno S, Ghebremichael MS, Allen TM, Tager AM, Burton DR, Luster AD. Protection of humanized mice from repeated intravaginal HIV challenge by passive immunization: a model for studying the efficacy of neutralizing antibodies in vivo. J Infect Dis. 2016:214:612-6.

34. Taylor BM, Foulke JS, Flinko R, et al. An alternation of human immunodeficiency virus gp41 leads to reduced CCR5 dependence and CD4 independence. J Virol. 2008;82:5460-71.

35. McCullough LD, de Vries GJ, Miller VM, et al. NIH initiative to balance sex of animals in preclinical studies: generative questions to guide policy, implementation, and metrics. Biol Sex Differ. 2014;5:15.

36. Nischang M, Sutmuller R, Gers-Huber G, et al. Humanized mice recapitulate key features of HIV-1 infection: a novel concept using long-acting anti-retroviral drugs for treating HIV-1. PLoS ONE. 2012;7(6): e38853. https://doi.org/10.1371/journal.pone.0038853.

37. Heredia A, Hassounah S, Medina-Moreno S, et al. Monotherapy with either dolutegravir or raltegravir fails to durably suppress HIV viraemia in humanized mice. J Antimicrob Chemother. 2017;72(9):2570-3. https:// doi.org/10.1093/jac/dkx195.

38. Weichseldorfer W, Affram Y, Heredia A, et al. Anti-HIV activity of standard CART in primary cells is intensified by CCR5-targeting drugs. AIDS Res Hum Retrovir. 2020;36(10):835-41.

39. Pallikkuth S, Sharkey M, Pahwa S, et al. Peripheral T follicular helper cells are the major HIV reservoir within central memory CD4 T cells in peripheral blood from chronically HIV-infected individuals on combination antiretroviral therapy. J Virol. 2016;90(6):2718-28.

40. Lee GQ, Orlova-Fink N, Einkauf K, et al. Clonal expansion of genomeintact HIV-1 in functionally polarized Th1 CD4 ${ }^{+}$T cells. J Clin Invest. 2017;127(7):2689-96. https://doi.org/10.1172/JC193289.

41. Tebas P, Kumar P, Hicks C, et al. Greater change in bone turnover markers for efavirenz/emtricitabine/tenofovir disoproxil fumarate versus dolutegravir + abacavir/lamivudine in antiretroviral therapy-naive adults over 144 weeks. AIDS. 2015;29:2459-64.

42. Acosta RK, Willkom M, Andreatta K, et al. Switching to bictegravir/emtricitabine/tenofovir alafenamide (B/F/TAF) from dolutegravir (DTG)+F/TAF or DTG+F/tenofovir disoproxil fumarate (TDF) in the presence of preexisting NRTI resistance. J Acquir Immune Defic Syndr. 2020;85(3):363-71.

43. Garcia-Vadal E, Castelvi M, Pujantell M, et al. Evaluation of the innate immune modulator acitretin as a strategy to clear the HIV reservoir. Antimicrob Agents Chemother. 2017:61:e1368-411.

44. Cihlar T, Fordyce M. Current status and prospects of HIV treatment. Curr Opin Virol. 2016;18:50-6.

45. Harder BG, Blomquist MR, Wang J, et al. Development in blood-brain barrier penetrance and drug repurposing for improved treatment of glioblastoma. Front Oncol. 2018;8:462.

46. Coiras M, López-Huertas MR, Pérez-Olmeda M, Alcamí J. Understanding HIV-1 latency provides clues for the eradication of long-term reservoirs. Nat Rev Microbiol. 2009;7(11):798-812.

47. Ventura JD, Beloor J, Haugh KA, et al. Longitudinal bioluminescent imaging of HIV-1 infection during antiretroviral therapy and treatment interruption in humanized mice. PloS Pathog. 2019:15(12):e1008161.

48. Satou Y, Katsuya H, Fukuda A, et al. Dynamics and mechanisms of clonal expansion of HIV-1-infected cells in a humanized mouse model. Sci Rep. 2017;7:6913.

\section{Publisher's Note}

Springer Nature remains neutral with regard to jurisdictional claims in published maps and institutional affiliations. 